\title{
Characteristics of the terrestrial field-aligned current system
}

\author{
J. W. Gjerloev ${ }^{1,4}$, S. Ohtani ${ }^{1}$, T. Iijima ${ }^{2}$, B. Anderson ${ }^{1}$, J. Slavin ${ }^{3}$, and G. Le ${ }^{3}$ \\ ${ }^{1}$ Applied Physics Laboratory, Johns Hopkins University, Laurel, MD 20723, USA \\ ${ }^{2}$ Nerima-ku, Tokyo, Japan \\ ${ }^{3}$ NASA-GSFC, Greenbelt, MD, USA \\ ${ }^{4}$ Department of Physics and Technology, University of Bergen, 5007, Norway
}

Received: 7 March 2011 - Revised: 14 September 2011 - Accepted: 24 September 2011 - Published: 4 October 2011

\begin{abstract}
We present the first ever comprehensive statistical study of the spatiotemporal characteristics of field-aligned currents in the terrestrial magnetosphere-ionosphere system using multi point measurements. We determine how the FAC density, variability and scale size are coupled. The three ST 5 satellites were in a pearls-on-a-string formation making measurements of the magnetic field with variable interspacecraft separations ranging from a few seconds to about $10 \mathrm{~min}$. More than 4700 sets of satellite passes are analyzed using a robust correlation analysis aimed at determining the variability of the FAC system as a function of scale size and satellite spacing. We find significant differences between the FAC characteristics on the dayside and on the nightside in terms of dynamics of the current systems. On the dayside the FAC characteristics are found to be independent of IMF $B_{\mathrm{Z}}$ and geomagnetic activity while the nightside indicates increased variability during disturbed conditions. The boundary separating highly and poorly correlated FACs can be fitted by a linear line for satellite separations shorter than $60 \mathrm{~s}$ (dayside) and $160 \mathrm{~s}$ (nightside). We interpret this as the dayside and nightside magnetospheric reconfiguration times respectively. For times exceeding this the FAC characteristics are suggested to be controlled by the solar wind (dayside) and plasma sheet (nightside) dynamics. Finally, the characteristics of FAC system with scale sizes larger than $\sim 200 \mathrm{~km}$ (at ionospheric altitude) appear to be stable and repeatable on time scales of the order of a minute (i.e. comparable to the low-altitude orbiting satellite's traverse time across the auroral belt). In this sense, our results effectively validate the Iijima and Potemra (1978) assumption that on average the large-scale currents with scale sizes of the Region1 and
\end{abstract}

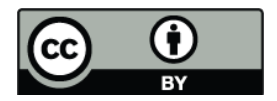

Correspondence to: J. W. Gjerloev (jesper.gjerloev@ift.uib.no)
Region2 are quasi-persistently significant in the transport of energy and momentum between the magnetosphere and the ionosphere.

Keywords. Ionosphere (Electric fields and currents) Magnetospheric physics (Current systems; Magnetosphereionosphere interactions)

\section{Introduction}

Field-aligned electrical currents (FACs) are the dominant process by which energy and momentum are transported from the magnetosphere to the ionosphere-thermosphere system (e.g. Foster et al., 1983; Lu et al., 1998). FACs flow between the magnetosphere and the ionosphere at all times and are strictly conserved between the low altitude ionosphere and the high altitude magnetosphere thus providing a unique opportunity to gain insight into the underlying magnetospheric processes (e.g. Iijima, 2000). In fact the importance of the FACs for the magnetospheric-ionospheric coupling was realized as early as the beginning of the last century by Birkeland (1908). Studies of FACs have been conducted by direct measurements from rockets and satellites and indirect measurements using e.g. ground based magnetometers. The importance of FACs for our understanding of ionospheremagnetosphere-solar wind coupling and interaction has naturally led to a vast number of studies and a number of review papers (e.g. Anderson and Vondrak, 1975; Kamide, 1982; Christiansen et al., 2002; Juusola et al., 2009).

Zmuda et al. $(1966,1967)$ published the first direct evidence of their existence and later the average configuration of the field-aligned currents was determined (Zmuda and Armstrong, 1974; Rostoker et al., 1975; Iijima and Potemra, 1978). In these groundbreaking statistical studies the FAC

Published by Copernicus Publications on behalf of the European Geosciences Union. 


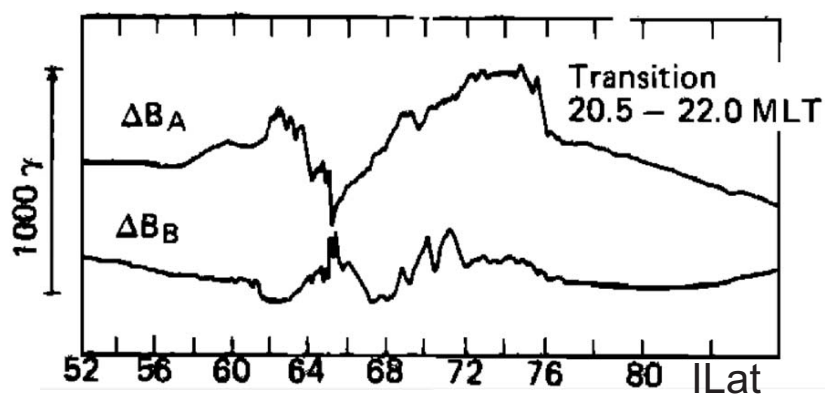

Fig. 1. Examples of Triad magnetometer data from 23 August 1974, at 07:47 UT (from Iijima and Potemra, 1978). The event occurred when $\mathrm{AL}$ was $-350 \mathrm{nT}$. The $\Delta B_{A, B}$ components indicate directions approximately east-west and north-south, respectively.

distribution was determined from magnetic field perturbations measured by the Triad satellite. It was found that the upward and downward FAC system consisted of two layers shaped as two approximately concentric circles with an overlap in the pre-midnight region. Their study and their conclusions, however, were based on three basic assumptions:

1. The observed magnetic field perturbations are due to static currents;

2. The currents are time independent over the time it takes for the satellite to cross the auroral oval (on the order of $5 \mathrm{~min})$;

3. Currents with scale sizes smaller than the Region 1 (R1) - Region 2 (R2) currents are insignificant.

The first two assumptions are both due to the inherent limitations of the data set that was available to the authors. The measurements made by a low Earth orbit satellite are separated in both time and space and hence it is not possible to uniquely determine if the measured perturbations are due to static currents or temporal variations. Typically the measured perturbations are assumed to be due to static currents and a simple FAC geometry which allow a calculation of the current density. Likewise, one must assume that the current system is constant over the time it takes the satellite to cross it. These assumptions seem reasonable but in fact they have never been addressed by any comprehensive statistical study. This serious shortcoming is due to the fact that the required data set has not been available before the launch of the ST 5 mission. The third assumption is likewise unsupported by the otherwise rich FAC literature. Figure 1 shows a satellite pass from the above mentioned Iijima and Potemra study. The magnetic field perturbations are clearly covering all scale sizes from the resolution of the data to the largescale R1-R2 sheets. Within these sheets we find smaller currents flowing anti-parallel to the sheet itself. We do not currently know if these smaller currents play any significant role in the total current flowing between the magnetosphere and the ionosphere. Neither do we know if they can be assumed to be static.

In stark contrast to the massive amount of single satellite data only a very limited number of multi-point satellite observations exist (e.g. The Auroral Turbulence II sounding rocket mission, Lynch et al., 1999; the Enstrophy sounding rocket mission, Zheng et al., 2003; and the CLUSTER II mission, Escoubet et al., 2001). However, rocket observations are brief and event based while the calculation and interpretation of currents deduced from Cluster observations is highly complicated by the orbit configuration. The Science and Technology 5 (ST 5) mission is so far the most comprehensive multi-point data set of magnetic field perturbations collected from LEO satellites. Slavin et al. (2008) published initial results from the ST 5 mission. They showed two events which appear to indicate that:

1. The FAC density is highly structured;

2. The FAC density changes significantly over the $1-6 \mathrm{~min}$ separation of the ST 5 satellites.

Thus, at least for these two events the multipoint measurements indicate that the FACs is highly structured in both space and time.

Thus, we find that although a rich literature exists on the FAC's the conclusions are based on fundamental assumptions that have not been tested. Single satellite measurements cannot distinguish between spatial and temporal variations, hence leading to the common questionable assumption that FACs are static (constant in time). As a consequence we currently know virtually nothing about the dynamics of the FACs. This study will, for the first time, provide a solid observational basis that allows us to test these assumptions by investigating the stability/invariability of the FAC system. The actual morphology of FACs is outside of our present aim.

In Sect. 2 we describe the data used; Sect. 3 outlines the technique; in Sect. 4 we show 12 typical events; Sect. 5 show statistical results; in Sect. 6 we discuss our results; and finally in Sect. 7 we summarize and draw conclusions.

\section{Data}

The three Space Technology 5 (ST 5) spacecraft were launched into a dawn-dusk, $105.6 \mathrm{deg}$ inclination, 300 by $4500 \mathrm{~km}$ orbit with a period of $136 \mathrm{~min}$. The ST 5 spacecraft were spin-stabilized with periods near $3 \mathrm{~s}$. The satellites were maintained in a "pearls-on-a-string" constellation (satellites are in same orbit plane but separated along the trajectory) with variable along track satellite separation providing a rich data set of multi-point measurements obtained with tri-axial fluxgate magnetometers carried by each of the three spacecraft. The satellite separation varied from a few seconds to about $10 \mathrm{~min}$. The launch date was 22 March 2006. In this study we utilize the entire magnetometer database obtained 

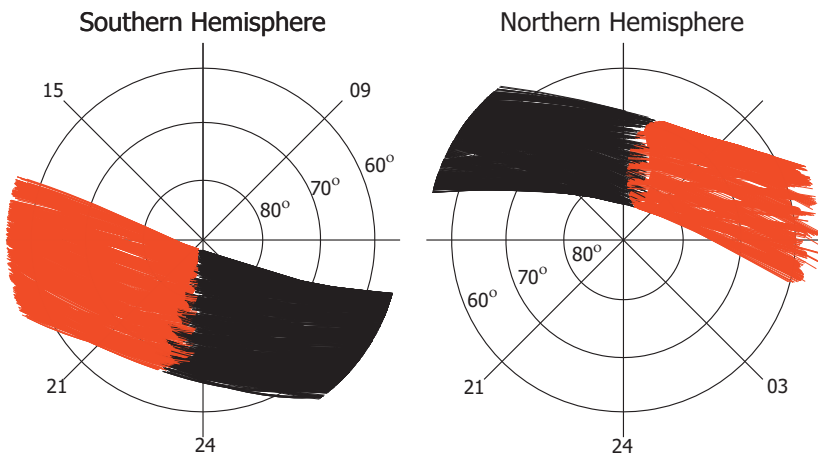

Fig. 2. Distribution of passes in each hemisphere used in this study. Black indicates poleward moving while red indicates equatorward moving. Magnetic local time and magnetic latitude is indicated.

by all three spacecraft during their 3 months lifetime (for a brief description of instrument performance see Slavin et al., 2008).

We use ACE observations for the solar wind conditions. Solar Wind data has been propagated to the front of the magnetosphere (courtesy J. Weygand) using the pseudominimum variance technique of Weimer et al. (2003) and Weimer (2004). Finally, AE indices are obtained from the World Data Center for Geomagnetism, Kyoto (http://wdc. kugi.kyoto-u.ac.jp/index.html).

\section{Technique}

\subsection{Basic data handling}

Prior to the actual data analysis we perform a few basic steps:

1. Determine the invariant magnetic latitude and magnetic local time of the satellites;

2. Subtract a main field model (AACGM) from the measured field;

3. Convert magnetic field measurements from solarmagnetic coordinates to a maximum-medium-minimum variance coordinate system (only the maximum variance direction is used in this study; e.g. Song and Russell, 1999);

4. Identify satellite auroral oval crossings ( $55 \mathrm{deg}$ to maximum magnetic latitude or maximum latitude to $55 \mathrm{deg}$ );

5. Identify sets of satellite passes (that is combinations of spacecraft: SC1-SC2; SC1-SC3; SC2-SC3) for which both satellite crossings are without data-gaps;

6. For each set of satellite passes determine the satellite separation in seconds.
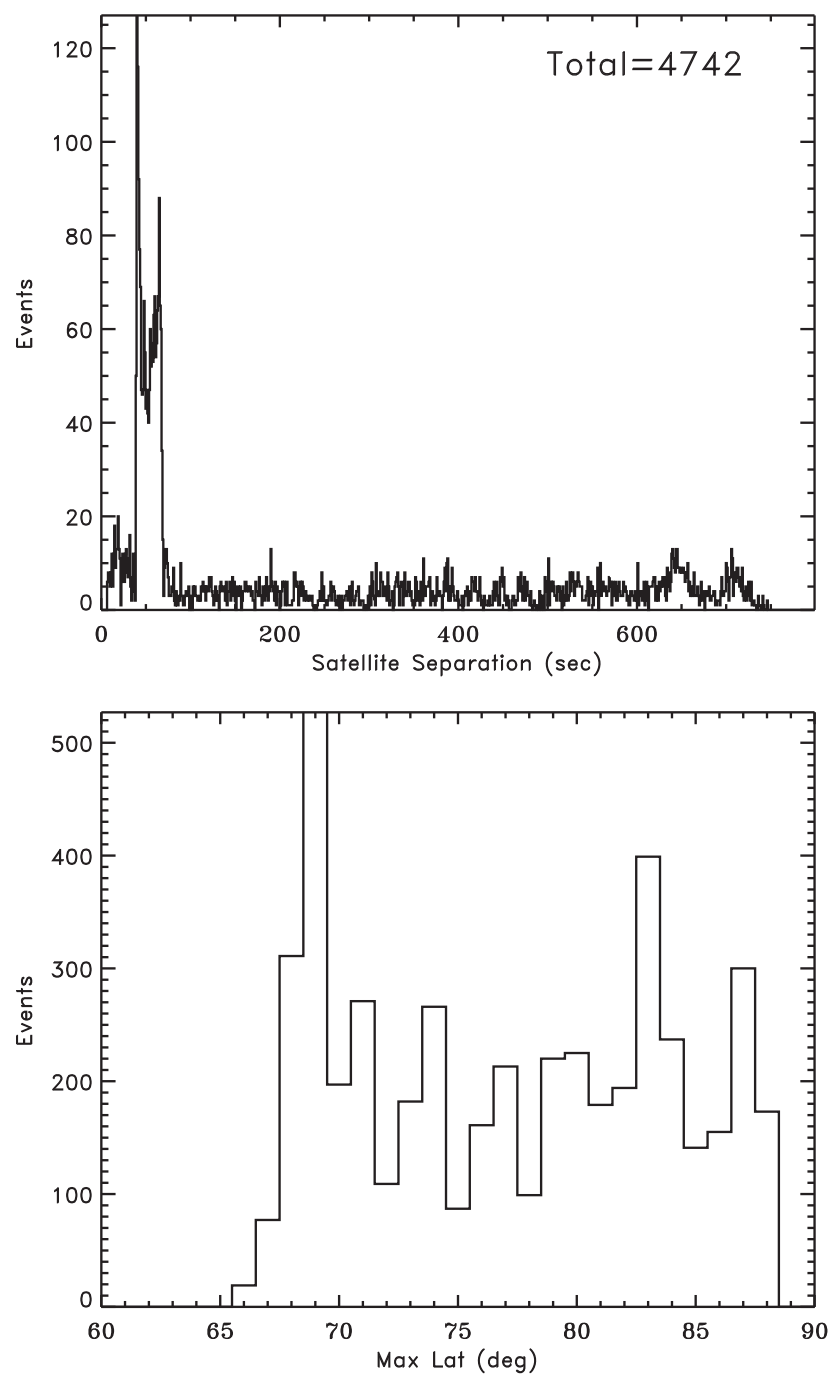

Fig. 3. Top: number of events as a function of satellite separation in seconds. Bottom: distribution of the maximum invariant latitude of the pass.

Using this basic approach we identified 4742 sets of satellite crossings with separations between $\sim 7 \mathrm{~s}$ and $\sim 750 \mathrm{~s}$. We utilize the entire database including passes in both hemispheres (Fig. 2). We refer to these sets of satellite crossings as "events". We show in Fig. 3 (top panel) the distribution of the 4742 events as a function of satellite spacing. Note the non uniform distribution with a large number of events centered around $50 \mathrm{~s}$ while the rest of the distribution appear to fluctuate around 5 events per $1 \mathrm{~s}$ separation. As mentioned in the "Data" section the ST 5 satellites were inserted into a dawn-dusk, $105.6 \mathrm{deg}$ inclination, $300-4500 \mathrm{~km}$ orbit. The orbit inclination combined with the offset of the magnetic pole results in orbits for which the satellites only skimmed the auroral oval rather than crossing it. The distribution of the maximum latitude during a pass is shown in bottom panel of Fig. 3. 
Regarding point (2) we determine the maximum variance direction for the leading satellite and use the same matrix for the trailing. This is the only way we can determine the variability, $d / d t$, as the paper is focusing on.

\subsection{Statistical approach}

To determine how the variability, intensity and scale-size of FACs are coupled we perform a simple yet robust correlation analysis. This is a three steps process:

1. Use a sweeping narrow band-pass filter (Hanning) on each of the two SC data sets to determine the correlation coefficient between the two filtered data sets as a function of frequency $C=C(f)$. The amplitude $A=A(f)$ is determined from the complex spectrum as the square root of the power;

2. Combine all events (sets of passes with varying satellite separation) to determine $C=C(f, \Delta T)$ where $\Delta T$ is the satellite separation in seconds;

3. Convert frequency to FAC scale size, $S$, to determine $C=C(S, \Delta T)$.

While the first two steps should be self explanatory the third step needs some explanation. Our analysis is complicated by the eccentricity of the orbit $(300-4500 \mathrm{~km})$. This results in significant differences between the pass duration. For the Northern Hemisphere perigee passes the duration are typically $\sim 500 \mathrm{~s}$ while for the Southern Hemisphere apogee passes the duration are typically $\sim 1500 \mathrm{~s}$. Taking the variable pass duration into account we calculate the scale size as:

$S=0.5 \cdot D /(f \cdot T)$

where $D$ is the spherical distance at ionospheric altitude of the satellite pass in $\mathrm{km}, T$ is the duration of the pass in $\mathrm{s}$, and $f$ is in $\mathrm{Hz}$. The factor 0.5 is a conversion factor assuming that the FAC scale size is half a full period. Thus, we determine $C=C(S, \Delta T)$.

\subsection{A note on current density calculation}

We do not convert the measured magnetic field perturbations from nT to current density. Deducing the current density from single satellite observations is based on a list of simplistic assumptions (for example infinite current sheets and that the measured magnetic field perturbations are due to static currents). Most often one or more of these assumptions are violated (e.g. Hoffman et al., 1994) thereby producing erroneous current densities. This is particularly important for present study since any of the assumptions on which the current density calculations are based on could be a function of either scale size or variability. For example, we could hypothesize that large scale current segments are more likely to fulfill the infinite current sheet assumption than small scale sizes. If this is the case, using current densities would introduce a crippling bias in our 2-D correlation analysis. By using the measured perturbations we do not introduce a possible error source. We can, however, straightforwardly interpret the statistical characteristics of the magnetic field perturbations as reflecting the statistical characteristics of the FACs since these are the cause of the measured magnetic field perturbations as described by Ampere's law. Finally, using the so-called 1-D curlometer technique (e.g. Sugiura, 1984; Luhr et al., 1994; Anderson et al., 2000) to calculate the instantaneous current density between two spacecraft eliminates the $d / d t=0$ assumption but it introduces another assumption constant current density between the two spacecraft.

\section{Typical events}

This section is intended to illustrate the technique outlined in the previous section as well as to show some typical events supporting the statistical results (Sects. 5 and 6). For three different satellite separations (approximately $15 \mathrm{~s}, 60 \mathrm{~s}$ and 600 s) we show four passes: a dusk and a dawn pass from each hemisphere. We choose to show 12 typical events to illustrate the richness of the data set which includes more than 4700 events.

Figure 4 shows 4 events with very short satellite separations ranging from $11 \mathrm{~s}$ to $18 \mathrm{~s}$. The panels show the measured perturbations (maximum variance direction) for each satellite as well as the scale-size dependent correlation $(C(S))$ calculated from the two data sets. The top panel shows two large-scale field aligned current segments with some smaller scale currents superposed. The two sets of observations (for example from satellite set SC1-SC2) closely repeat each other and only a close inspection of the two traces indicate minor differences. The correlation analysis support this finding. For scale sizes larger than about $50 \mathrm{~km}$ the correlation is close to unity. For smaller scale sizes, however, the two sets of observations are poorly correlated. The second panel shows a dusk event which is in good agreement with the previous event. The two Southern Hemisphere events show more complex current configurations with numerous up and down flowing current segments. This apparent complexity, however, does not change the results of the correlation analysis. In agreement with the two Northern Hemisphere passes we find the two data sets to be highly correlated for scale sizes larger than $\sim 50 \mathrm{~km}$. Thus, these four passes all indicate that current filaments with scale sizes larger than $\sim 50 \mathrm{~km}$ change on time scales longer than the $11-18 \mathrm{~s}$ by which these passes were separated.

For satellite separations of about $60 \mathrm{~s}$ we likewise show 4 events (Fig. 5). In comparing with the previous examples larger differences between the two satellites are apparent. In all events the correlation analysis indicates significant changes to current filaments with scale sizes of about $200 \mathrm{~km}$ or smaller. Thus, we find current filaments with scale sizes 

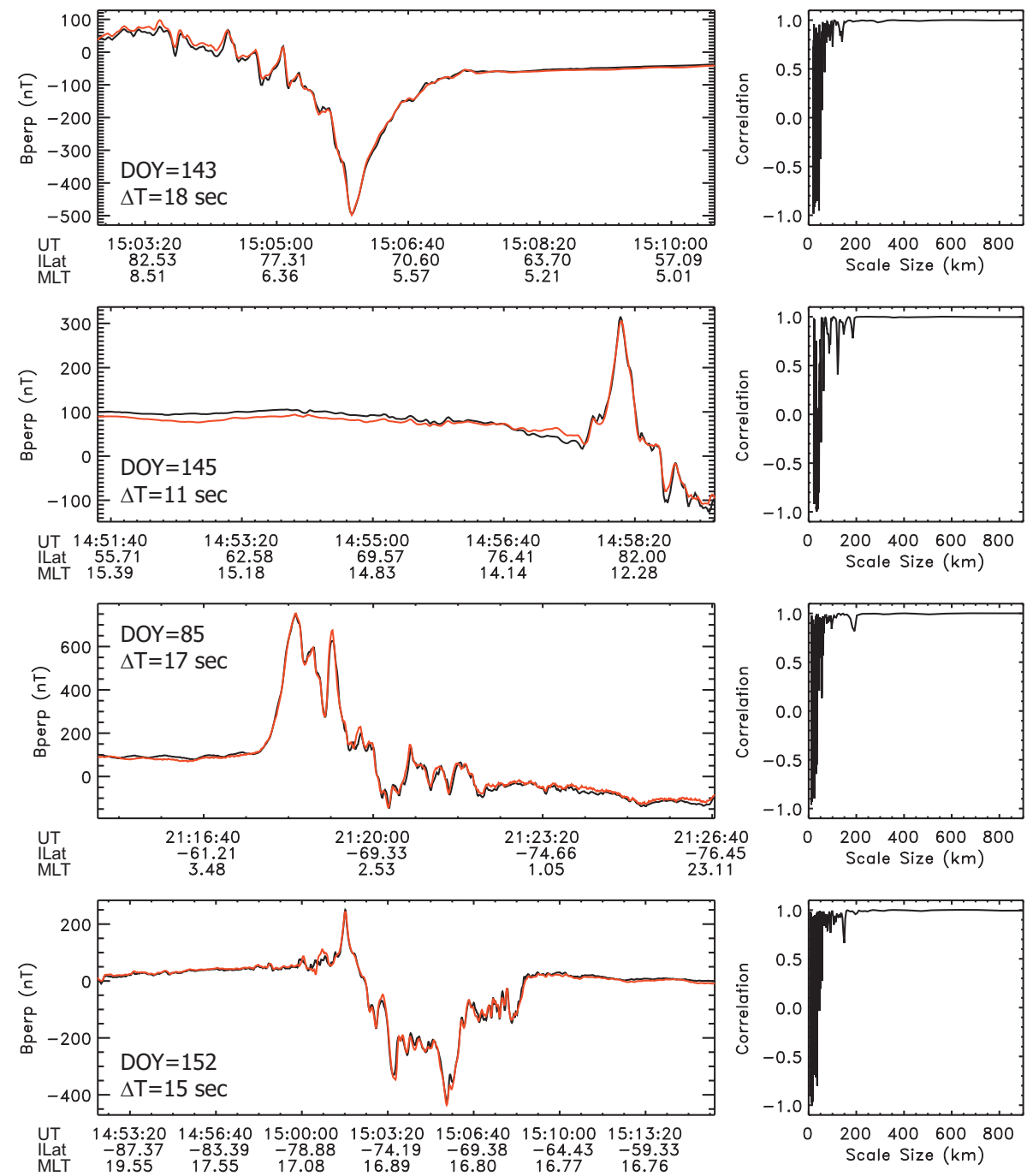

Fig. 4. Left panels: magnetic field perturbations measured by two different spacecraft (red/black) during four typical events with satellite separations around $15 \mathrm{~s} . \Delta T$ indicate the satellite separation in seconds and DOY is the day-of-year. Right panels: Scale size dependent correlation coefficient. Note the similarity of the two data sets supported by the correlation analysis approaching unity for scale sizes longer than about $50 \mathrm{~km}$. Top/bottom panels are for Northern/Southern Hemisphere passes.

smaller than roughly $200 \mathrm{~km}$ vary on time scales of about $60 \mathrm{~s}$ or less.

In the final examples we show four events with satellite separations of about $10 \mathrm{~min}$ (Fig. 6). The top panel shows an event where the two data sets show significant differences. The correlation analysis indicates poor correlation up to about $700 \mathrm{~km}$. Similar results are seen in the three other examples with significant changes to even the largescale current sheets. The third panel show an event where a large-scale current sheet has developed during the $612 \mathrm{~s} \mathrm{sep}-$ arating the two satellites. Thus, on time scales of $10 \mathrm{~min}$ we find significant changes to the entire FAC system at all scale sizes.
In conclusion we find that these 12 events indicate that FAC scale size and variability is related. Larger current segments appear to be less variable at all local times.

\section{Data base analysis}

We now show the statistical results based on the entire database. For each event we have calculated the scale size dependent correlation, $C=C(S)$, and by combining all passes with variable satellite separation, $\Delta T$, we can deduce the 2dimensional correlation distribution $C=C(S, \Delta T)$ (Fig. 7). We have used a simple boxcar smoothing ( $3 \mathrm{~s}$ window) for satellite separations greater than $70 \mathrm{~s}$ where the event coverage is only averaging about 5 events per second (see Fig. 3). 

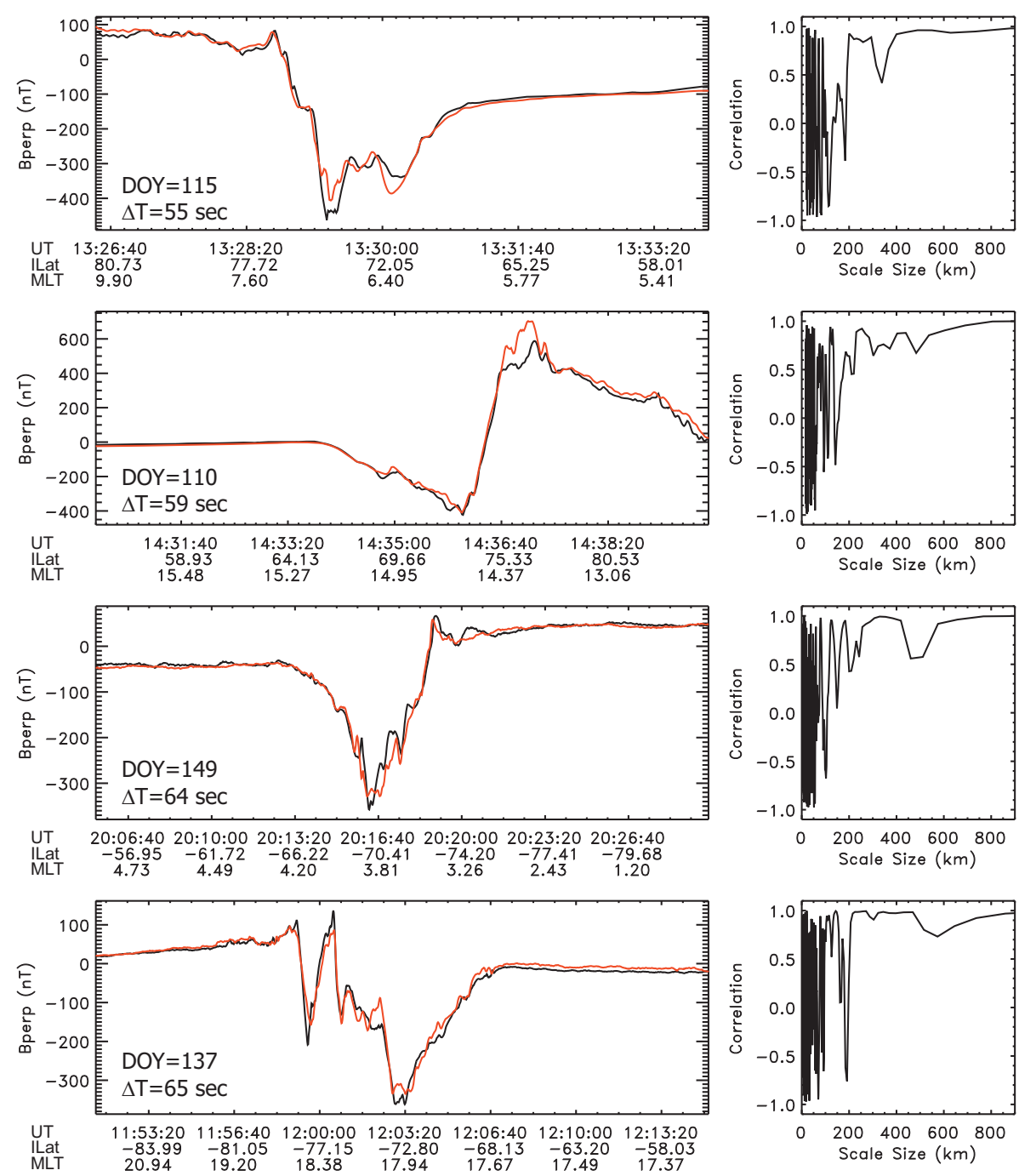

Fig. 5. Same as Fig. 4 but for satellite separations around $60 \mathrm{~s}$.

It should be noted that since all events are included in deducing the figure we effectively make the assumption that the characteristics of the FAC system are independent of geomagnetic conditions, local time, IMF $B_{Z}$ or any other parameter that may vary from event to event. For example, by including all satellite observations we assume that the scale size dependent variability of the currents is independent on the solar wind conditions, i.e. we assume the characterization of the magnetosphere-ionosphere FAC system is maintained from event to event. Thus, it is a repeatable system. It remains to be shown that this is a valid assumption.

The plots of the correlation as a function of scale size and satellite separation show fairly well organized regions of high correlation and of low correlation (Fig. 7, left panel). For small scale sizes and large satellite separation the two sets of observations are uncorrelated while short satellite separation and large scale sizes are highly correlated. We can quantify this by identifying the location of the boundary (correla- tion $=0.5$ ) between these two regions (right panel). The black dots appear to fit a straight line for satellite separations less than about $140 \mathrm{~s}$. The scatter increases for larger separations and the slope of the linear line appear to be steeper. Simple linear fitting suggests that the boundary between highly correlated and poorly correlated can be approximated by:

$$
\begin{aligned}
f(S) & =\left\{\begin{array}{c}
0.35 \cdot S, S \leq 400 \mathrm{~km} \\
1.50 \cdot S-450, S>400 \mathrm{~km}
\end{array}, \quad\right. \text { or } \\
g(\Delta T) & =\left\{\begin{array}{c}
2.73 \cdot \Delta T, \Delta T \leq 140 \mathrm{~s} \\
0.67 \cdot \Delta T+300, \Delta T>140 \mathrm{~s}
\end{array}\right.
\end{aligned}
$$

where $S$ is the scale size and $\Delta T$ is the satellite separation. Using this simple relationship we find that for satellite separations of 15/60/600 s only current segments with scale sizes larger than $\sim 40 / 165 / 700 \mathrm{~km}$ are correlated. This is in fair agreement with the typical events shown in the previous paragraph. Naturally, the value of 0.5 is an arbitrarily chosen value and one could argue that any other value may be more 

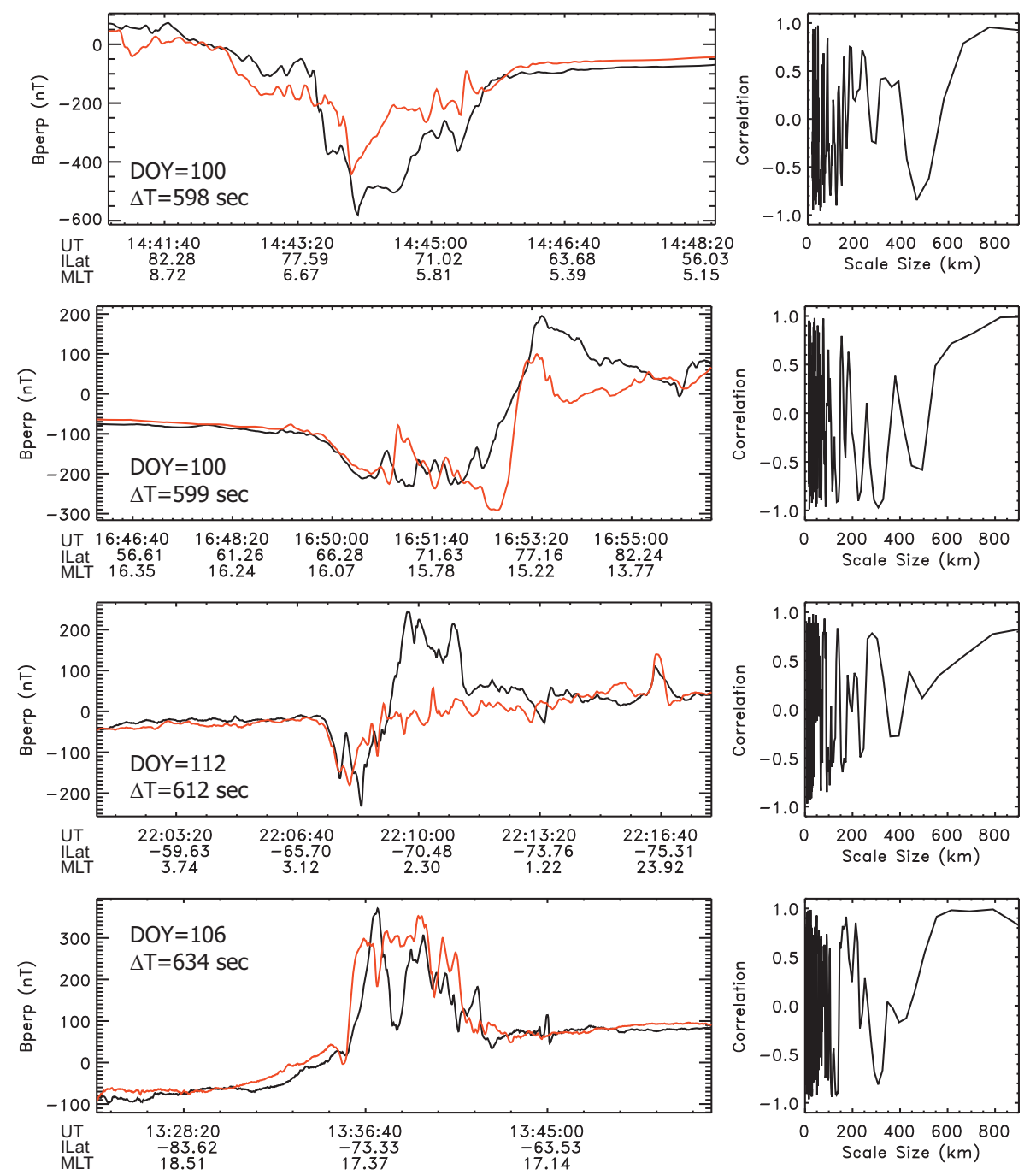

Fig. 6. Same as Fig. 4 but for satellite separations around $600 \mathrm{~s}$.

appropriate. The purpose, however, is simply to elucidate the location of the boundary between correlated and uncorrelated. A change to 0.6 simply results in a shift in the positive $\mathrm{x}$-axis direction without any noteworthy change in the slope. Finally, note that for separation times exceeding about $600 \mathrm{~s}$ the linear relationship is purely extrapolation as significant scatter is present. It is possible that this range should not be included in the fit.

In the range $\Delta E \in[40 \mathrm{~s}, 70 \mathrm{~s}]$ the event distribution has a sharp peak providing excellent statistics (see Fig. 3). This allows us to zoom into this area and increase the resolution of the 2-D correlation plot as can be seen in Fig. 8. The boundary between highly and poorly correlated is remarkably well defined. This plot as well as the plot in Fig. 7 (right panel) suggests that the FAC system with spatial scales of $\sim 200$ 400 (comparable to region 1 and region 2 currents) $\mathrm{km}$ can be described as static currents on time scales of 1 min or less.

\section{Discussion}

The surprisingly clear results of Fig. 7 supports the assumption that the magnetosphere-ionosphere system is indeed repeatable. The ST 5 data set gives us a glimpse of the physics, but to fully interpret our results we must address the inherent limitations of the data set and the technique. The four main limitations of the ST 5 data set are: (1) a sun-synchronous dawn-dusk orbit with no coverage of the noon-midnight sector; (2) orbital inclination of $>105 \mathrm{deg}$; (3) inherent limitations of a spin-stabilized spacecraft ( $3 \mathrm{~s} \mathrm{period})$; (4) $1 \mathrm{~s}$ resolution data used in this study; and (5) limited mission lifetime $\sim 90$ days.

Limitation (1) is unfortunate for studies of magnetosphereionosphere coupling as for example substorms tend to occur in the midnight region. Limitation (2) complicate current calculations since the satellite may not cross the auroral zone and hence the current density calculation technique 

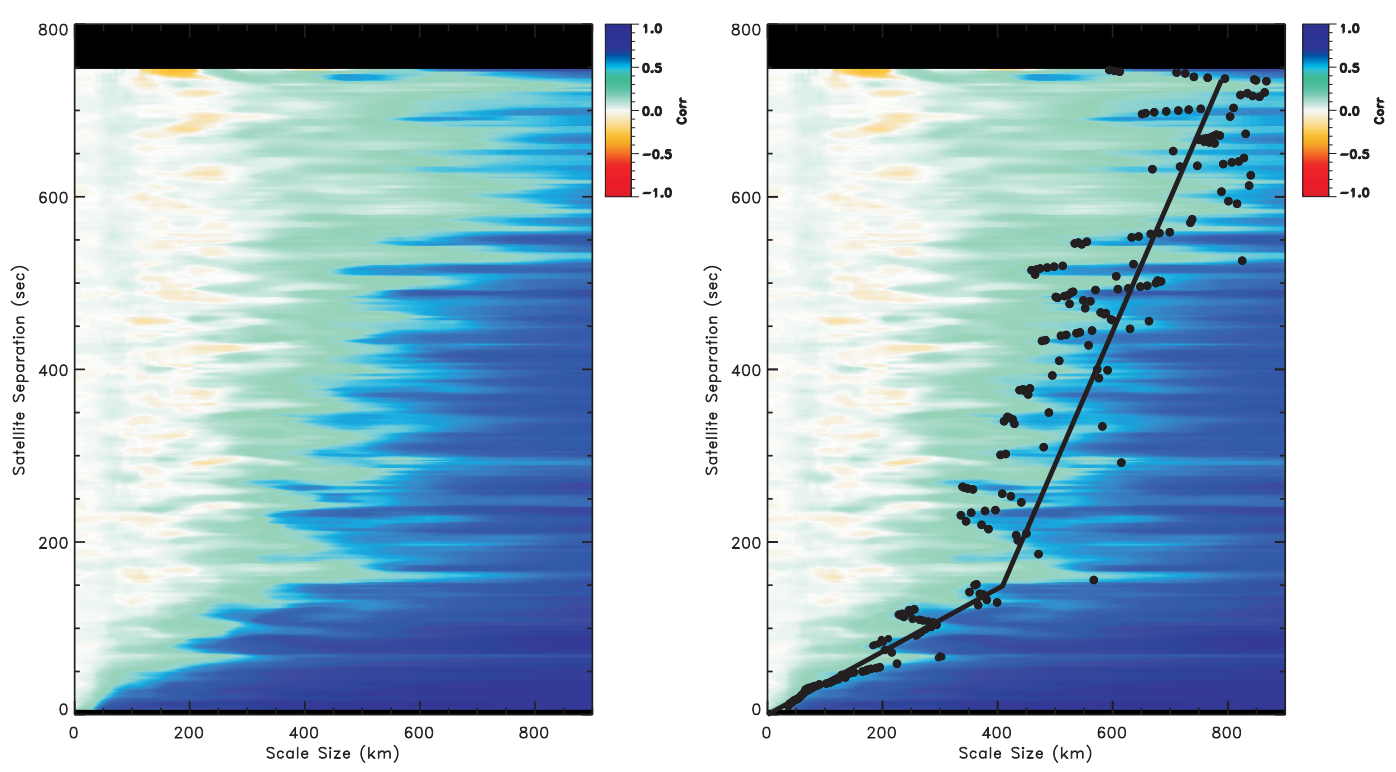

Fig. 7. Left panel: correlation as a function of scale size and satellite separation. Note the well organized regions of high and low correlation despite the database including dayside and nightside passes occurring during all types of geomagnetic conditions and solar wind conditions. Right panel: same as left panel but with correlation coefficients of 0.5 superposed (black dots). Linear lines are fitted to the dots indicating the location of the correlated/uncorrelated boundary.

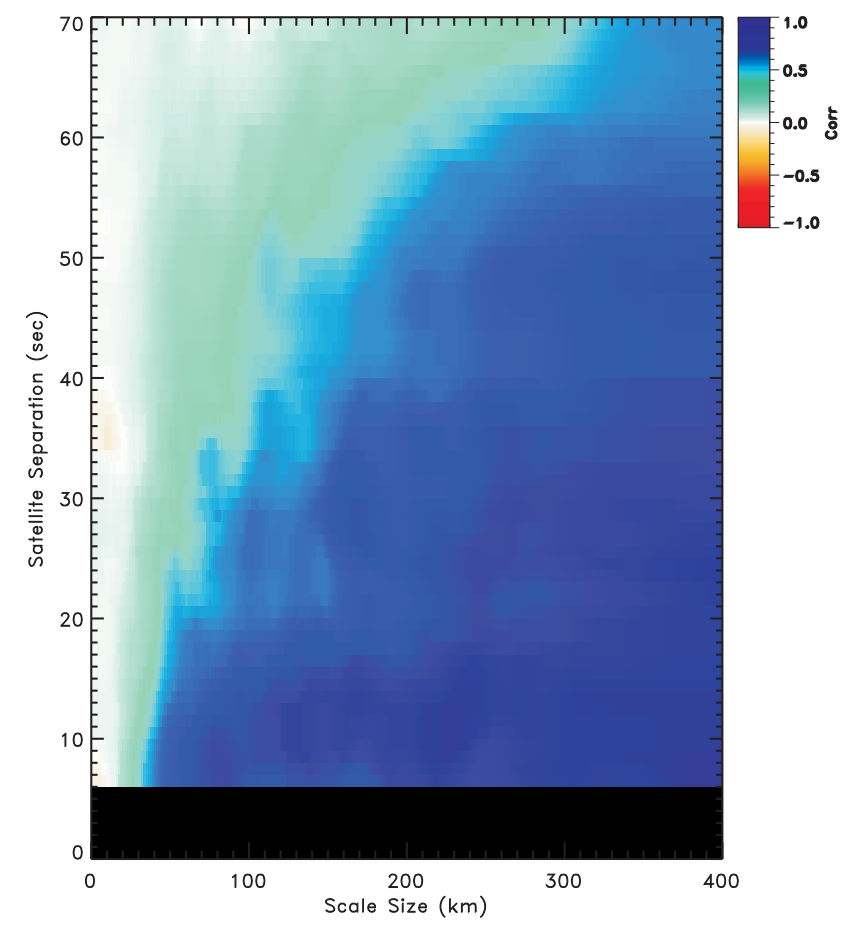

Fig. 8. Part of Fig. 7 for small scale sizes and satellite separations less than $70 \mathrm{~s}$.

(Sect. 3.3) is violated. We address this problem by not calculating the current density but only analyzing the measured magnetic field perturbations. Limitations (3) and (4) affect the range of scale sizes we are able to investigate. A satellite velocity of $7 \mathrm{~km} \mathrm{~s}^{-1}$ corresponds to a minimum scale size of $7 \mathrm{~km}$ but anything less than $\sim 20 \mathrm{~km}$ is questionable due to the spin period averaging. This, however, is only a rough measure of the minimum distance since we have to map the satellite position from its actual position to the ionospheric magnetic footpoint to calculate the relevant ionospheric velocity. Nevertheless, we caution making any conclusions regarding scale sizes smaller than $\sim 20 \mathrm{~km}$. The linear fit for satellite separations less than $140 \mathrm{~s}$ in Fig. 7 does pass through origin but this does not appear to be supported by Fig. 8. We claim this apparent error is due to limitations (3) and (4). Finally, limitation (5) prevent a study of seasonal effects and more detailed studies of, for example, the dependence on IMF $B_{\mathrm{Z}}$ (although we show some results below).

\subsection{Inherent assumptions}

A latitudinal shift of an otherwise static current system will affect our correlation analysis since a latitudinal shift results in a phase shift of the bandpass filtered signal. Thus, we have effectively estimated the frequency (or scale size) dependent total derivative of $d b / d t$ :

$\frac{d b}{d t}=\frac{\partial b}{\partial t}+\boldsymbol{U} \cdot \nabla b$

where $\boldsymbol{U}$ is the velocity of the current segments in the satellite reference frame. That is, the observed changes in $b$ can be due to the partial derivative with respect to time as well as any movement of the currents (producing $b$ ) that would occur in the time between the two satellite passes. Strictly we 


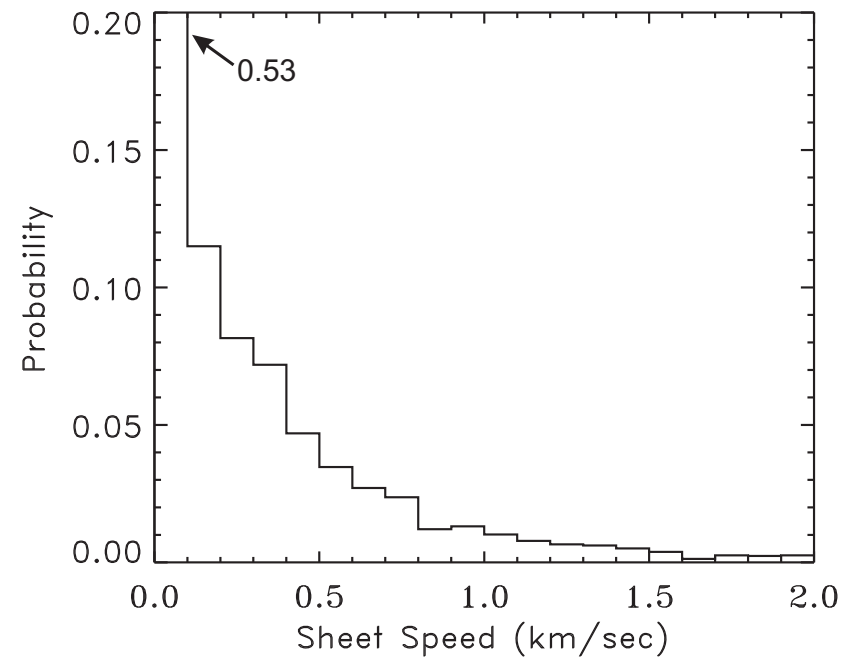

Fig. 9. Probability distribution of current region speed (along satellite track) determined from cross correlation analysis. The value 0.53 indicate that speeds of less than $100 \mathrm{~m} \mathrm{~s}^{-1}$ are seen in $53 \%$ of the events.

have no way of separating these terms in order to determine the cause of the observed $d b / d t$. Slavin et al. (2008) and Le et al. (2009) both estimated the velocity, $\boldsymbol{U}$, using cross correlation between the two sets of observations. Naturally, the data set only allows an estimation of the component of the velocity vector along the spacecraft trajectory (speed). Perhaps more importantly, however, is that a speed determination from cross correlating the two time series will be driven by the largest amplitude frequencies which are likely the large-scale size current segments (Sect. 6.2). As a consequence we have not attempted a separation of the two right side terms and have instead derived a measure of the total time derivative although we acknowledge that this may not be solely due to temporal variations.

The 12 typical events shown in Figs. 4-6 did not appear to indicate any significant shift of the large-scale current segments. With the above mentioned limitations we have determined the sheet speed distribution by cross correlating the two data sets for each event. We calculate the satellite speed after the appropriate mapping of their position to ionospheric altitudes (similar to Sect. 3) and determining the cross correlation lag in s. In more than $50 \%$ of the events we find sheet speeds to be less than $100 \mathrm{~m} \mathrm{~s}^{-1}$ (Fig. 9). Given the inherent ST 5 limitations a number of the orbits graze the auroral oval rather than crossing it and there is no reason to believe that the speed calculated for individual events (using the cross correlation analysis) provides any useful information regarding the velocity vector of the individual current sheets.

\subsection{Amplitude}

FACs of all scale-sizes play a role in the transfer of energy and momentum between the magnetosphere and the iono-

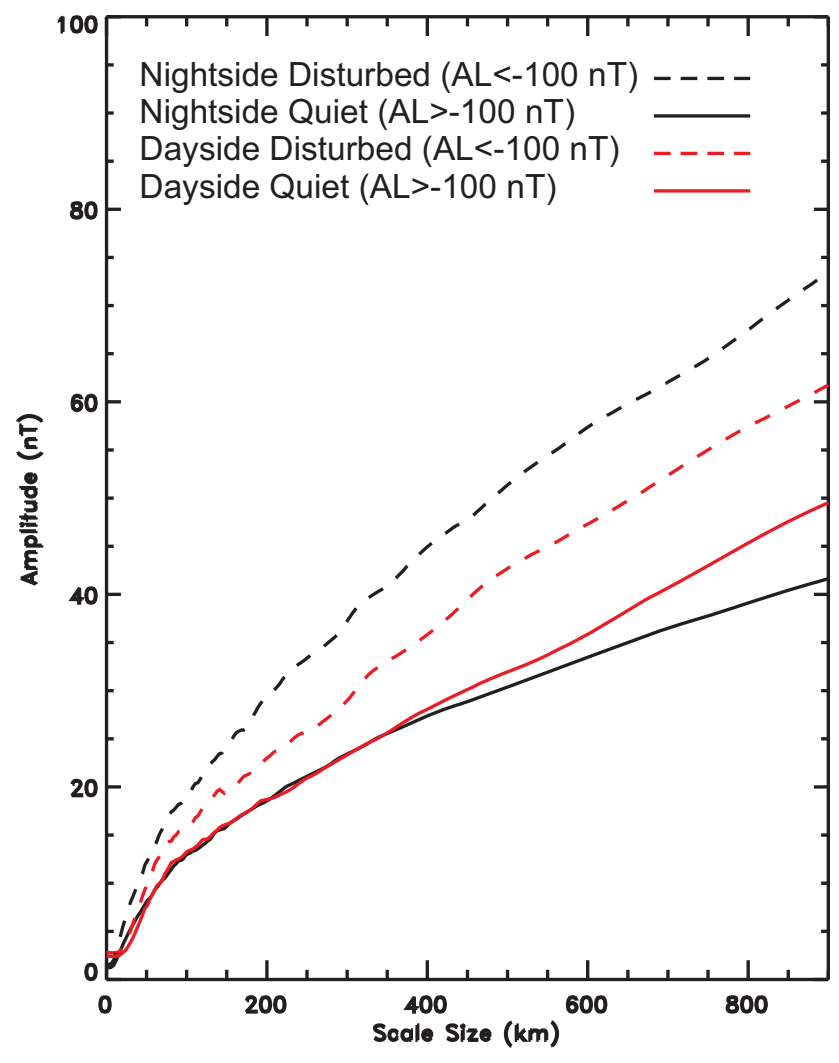

Fig. 10. Scale size dependent amplitude on the dayside/nightside for disturbed/undisturbed conditions as defined by $|\mathrm{AL}|>100 \mathrm{nT}$ and $|\mathrm{AL}| \leq 100 \mathrm{nT}$.

sphere. To understand the significance of the different FAC scale sizes in this magnetosphere-ionosphere coupling we determine the scale size dependent amplitude $A=A(S)$ from the complex spectrum as the square root of the power (also see Sect. 3). Because of the high eccentricity orbit we must scale the measured magnetic field perturbations (Ohtani et al., 1996):

$\Delta B_{\mathrm{a}}=\Delta B_{\mathrm{s}}\left(\frac{r_{\mathrm{s}}}{r_{\mathrm{a}}}\right)^{3 / 2}$

where $\Delta B_{\mathrm{a}, \mathrm{s}}$ are the measured field perturbations at the top of the atmosphere ( $200 \mathrm{~km}$ altitude) and at the altitude of the satellite respectively; and $r_{\mathrm{a}, \mathrm{s}}$ are the altitudes at the top of the atmosphere (200 km altitude) and the satellite, respectively.

We expect that $A$ is a function of activity but it also seems reasonable that there is a dayside/nightside dependence. In investigating this we are subject to the orbital configuration of the ST 5 mission. We have limited local time coverage and hence simplistically divide our passes into dayside passes (Northern Hemisphere) and nightside passes (Southern Hemisphere), respectively.

To investigate the amplitude dependence on geomagnetic activity we use the provisional AL index as a proxy. Active/quiet conditions are defined as $|\mathrm{AL}|>100 \mathrm{nT}$ and 


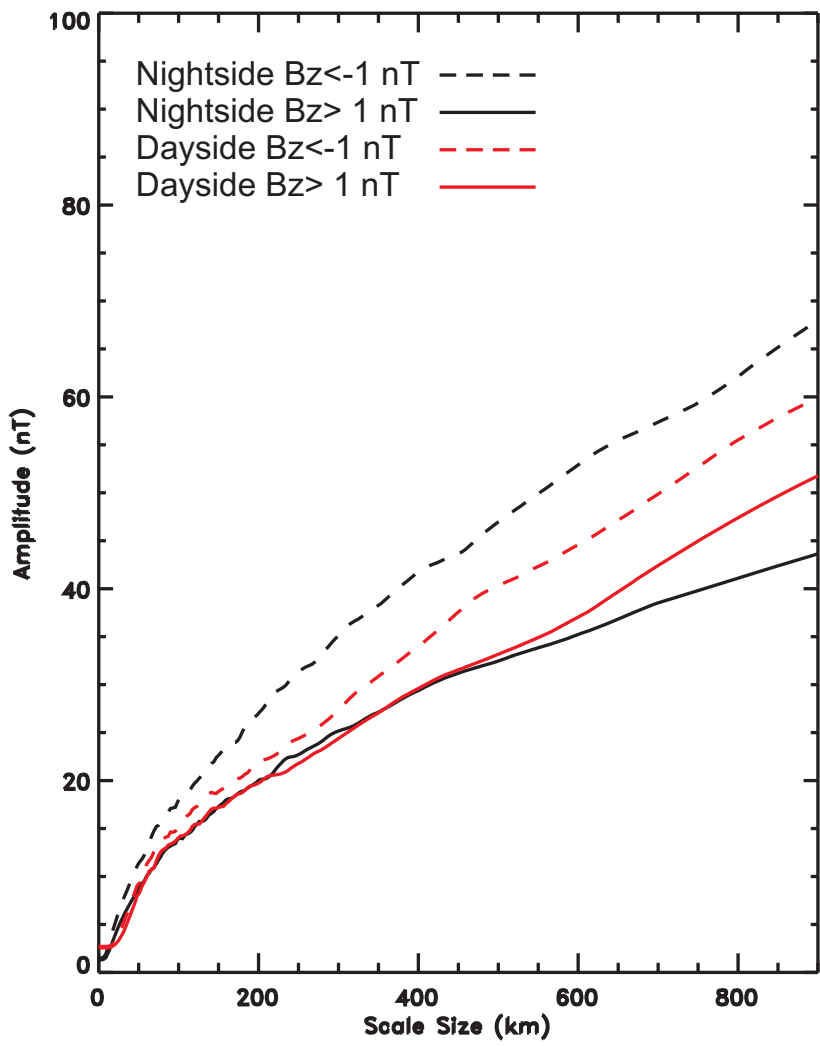

Fig. 11. Same as Fig. 10 but for negative and positive $B_{\mathrm{Z}}$ values.

$|\mathrm{AL}| \leq 100 \mathrm{nT}$, respectively. We acknowledge the inherent shortcomings of this index (e.g. Rostoker, 1972) and agree that the threshold of $-100 \mathrm{nT}$ is a fairly arbitrary limit. This threshold is driven by the need to have a sufficient number of events for the analysis. We argue, however, that for the purpose of investigating the effect of geomagnetic activity this approach is acceptable. Indeed, we find that the larger amplitudes are found on the nightside during disturbed conditions (Fig. 10). Modest enhancements is seen on the dayside while the amplitude of the FACs on the nightside on the other hand show a somewhat stronger dependence on AL. This is in good agreement with the Iijima and Potemra (1976) findings although a direct comparison between our results and theirs is complicated by the difference in technique. Nevertheless, we further find the quiet time dayside and nightside amplitudes to be virtually identical for scale sizes up to $\sim 400 \mathrm{~km}$ which is also in good agreement with their results.

Somewhat more unexpected we find an increase in the amplitude for all scale sizes. We interpret this as an indication that the magnetospheric substorm processes associated with the explosive release of energy stored in the magnetosphere spawn FACs covering basically all scale sizes. To determine the relative importance in the transport of the momentum and energy from the magnetosphere to the ionosphere we, however, note that the shorter scale sizes not only have smaller amplitudes but they are also more variable (Fig. 7).
We speculate that the dayside currents are either so-called directly driven by the solar wind IMF $B_{\mathrm{Z}}$ component and hence we determine $A=A\left(S, B_{\mathrm{Z}}\right)$. To account for a possible delay in the response of the FAC system to the IMF driver we have included IMF data from $10 \mathrm{~min}$ prior to the event. We organize the passes by the median of the IMF $B_{\mathrm{Z}}$ during the duration of the event as: $\operatorname{median}\left(B_{\mathrm{Z}}\right)<-1 \mathrm{nT}$ and median $\left(B_{\mathrm{Z}}\right)>1 \mathrm{nT}$. We realize that this simple binning method is not sufficient for a rigorous determination of $A=$ $A\left(S, B_{\mathrm{Z}}\right)$ but our database does not allow a more rigorous analysis. Figure 11 shows that the nightside FAC amplitude is a function of IMF $B_{\mathrm{Z}}$ but on the dayside the dependence is weak at best. Since substorm activity is strongly correlated with $B_{\mathrm{Z}}$ we expect that the nightside FACs are likewise strongly correlated with $B_{\mathrm{Z}}$ in agreement with the above findings (Fig. 10). This may lead us to suggest that the nightside plasma sheet that is presumably a source region of the nightside FAC system is controlled by the solar wind parameter of IMF $B_{\mathrm{Z}}$. By contraries, for the dayside FACs, a pronounced dependence on IMF $B_{\mathrm{Z}}$ is not discernible in apparent disagreement with Fig. 10 (dependence of substorm activity). FACs are always present on the dayside so while the morphology of the FAC system may change as a function of the IMF $B_{\mathrm{Z}}$ orientation the amplitude between the two sets of $B_{Z}$ conditions apparently remain fairly unaffected, indicative of a quasi-persistent solar wind source producing an offset amplitude.

As mentioned above we are limited by the orbital configuration of the ST 5 mission and our results are based upon observations with incomplete local time coverage. In the dark hemisphere both the ionospheric conductivity and the plasma density in the acceleration region are low, which presumably will result in intense auroral acceleration and intense FACs (Fujii and Iijima, 1987). Noteworthy, our results are in agreement with the findings of Iijima and Potemra (1976) who also found a distinct dependence on local time and activity.

\subsection{Controlling Parameters of the FAC system charac- teristics}

In deducing Figs. 7 and 8 we included all events: dayside/nightside, active/quiet conditions and all IMF conditions. With that in mind it seems remarkable that the pattern is consistent. In this paragraph we refine our analysis to determine the possible dependence of the above parameters.

In the Southern/Northern Hemisphere the crossings occurred on the nightside/dayside, respectively, allowing us to investigate differences between the FAC characteristics on the dayside and the nightside (Fig. 2). The ST 5 orbit provided nightside observations from the Southern Hemisphere and dayside observations from the Northern Hemisphere. We perform the above analysis for dayside and nightside events separately in order to determine possible differences in the FAC characteristics. Figure 12 shows the correlation plots for satellite separations in the $0-800 \mathrm{~s}$ range and the $0-70 \mathrm{~s}$ 

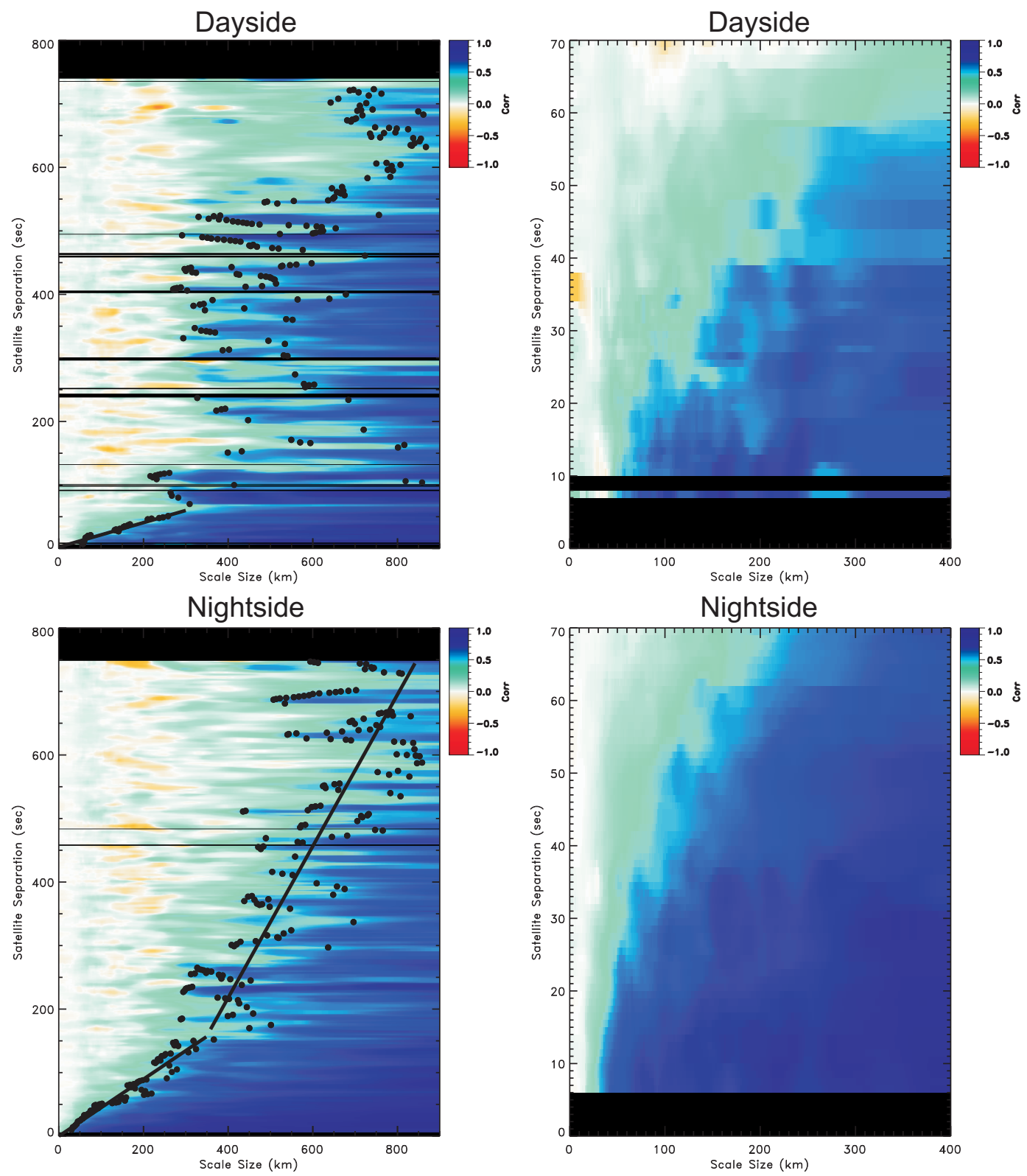

Fig. 12. Left panels: correlation as a function of scale size and satellite separation for dayside and nightside events, respectively. Black dots indicate correlations of 0.5 with linear lines superposed. Right panels: Part of left panel for small scale sizes and satellite separations less than $70 \mathrm{~s}$. The horizontal black bar on the dayside plot for separation time of 8-10 s is due to a lack of events in this interval.

range. In comparing the dayside and the nightside plots we note some striking differences:

1. Left column: On the nightside for separations exceeding $\sim 160$ s we find a fairly well defined linear separation boundary separating regions of high and low correlation. On the dayside, on the other hand, no such relationship appear to exist for separations exceeding $\sim 60 \mathrm{~s}$.

2. Left column: On the nighside for separations shorter than $\sim 160 \mathrm{~s}$ we find a linear separation boundary while on the dayside this only appear to exist up to separations of $\sim 60 \mathrm{~s}$. Further, there is a distinct difference in the slope between the dayside and nightside.

3. Right column: The highly correlated region (blue) appear significantly larger on the nightside than on the dayside. Thus, the FAC system appear to be more variable on the dayside than on the nightside. For example, with satellite separations of $40 \mathrm{~s}$ we find correlated FACs with scale sizes of $\sim 100 \mathrm{~km}$ while on the dayside correlations exceeding 0.5 require scale sizes to be $>200 \mathrm{~km}$. 
We approximate the location of the correlated/uncorrelated boundary as:

Dayside:

$$
\begin{aligned}
f(S) & =\left\{\begin{array}{c}
0.20 \cdot S, S \leq 300 \mathrm{~km} \\
\text { undetermined, } S>300 \mathrm{~km}
\end{array}, \quad\right. \text { or } \\
g(\Delta T) & =\left\{\begin{array}{c}
5.00 \cdot \Delta T, \Delta T \leq 60 \mathrm{~s} \\
\text { undetermined, } \Delta T>60 \mathrm{~s}
\end{array}\right.
\end{aligned}
$$

Nightside:

$$
\begin{aligned}
f(S) & =\left\{\begin{array}{c}
0.45 \cdot S, S \leq 350 \mathrm{~km} \\
1.20 \cdot S-260, S>350 \mathrm{~km}, \quad \text { or }
\end{array}\right. \\
g(\Delta T) & =\left\{\begin{array}{r}
2.22 \cdot \Delta T, \Delta T \leq 160 \mathrm{~s} \\
0.83 \cdot \Delta T+215, \Delta T>160 \mathrm{~s}
\end{array}\right.
\end{aligned}
$$

where the break points $(\sim 160 \mathrm{~s}$ on the nightside, and $\sim 60 \mathrm{~s}$ on the dayside) are approximate values. On the nightside for $\Delta T>600 \mathrm{~s}$ the linear relationship is primarily extrapolation as significant scatter in the data is present.

With the finding that dayside and nightside characteristics show pronounced differences we investigate a possible dependence on geomagnetic activity and IMF $B_{\mathrm{Z}}$ for the dayside and nightside separately. Only for satellite separations less than $70 \mathrm{~s}$ do we have a sufficient number of events to bin of the events. On the nightside we find that two linear fits are needed to fit the correlated/uncorrelated boundary: one for $\Delta T<160 \mathrm{~s}$ and another for $\Delta T>160 \mathrm{~s}$. While the limited statistics provides a likely explanation for the scatter seen in the latter interval the difference in the slopes is striking and cannot be explained by the event coverage. It is possible that better event coverage as a function of separation time (top panel of Fig. 2) would result in slightly different slopes but there is no reason to believe that this would affect any of our conclusions (e.g. the presence of a break around $160 \mathrm{~s}$ ). With the limitations of the data set in mind we simplistically determined the local time dependence as dayside/nightside. A more stringent determination of $C=C(S, \Delta T$, MLT) require additional observations that are not currently available.

To determine the dependence on geomagnetic activity we again use the provisional AL index as was done in the above Sect. 6.2. On the dayside we find little dependence on activity as seen in Fig. 13. There may be a hint of increased variability or poorer correlation for disturbed times but the dependence is weak at best. For the nightside, on the other hand, we find a clear dependence on activity with disturbed conditions being associated with more variability of the FACs. At first glance the difference between the characteristics on the dayside and nightside seems logical since AL during active times is typically defined by stations located on the nightside (e.g. Davis and Sugiura, 1966). However, we performed additional analysis using two bins of $\mathrm{AE}$ and AU to organize our data before deriving the 2-D distributions (similar to Fig. 13). No changes were found on the nightside. On the dayside we found that using AE/AU to organize the data led to slightly higher correlation than using AL. The meager improvement, however, may indicate that the dayside FACs characteristics is largely controlled by the solar wind conditions (also see below discussion). The lack of statistics, however, does not allow us to pursue this further.

Finally, we can determine the dependence on IMF conditions. If the FAC system is directly driven by the solar wind we expect a clear dependence on the IMF direction. We organize the events by the median IMF $B_{\mathrm{Z}}$ during the time of the event as $<B_{\mathrm{Z}}>>1 \mathrm{nT}$ and $<B_{\mathrm{Z}}><-1 \mathrm{nT}$. As for the amplitude analysis we take into account a possible IMF propagation delay by including data from $10 \mathrm{~min}$ prior of the event. Figure 14 shows that on the dayside we find little variations between northward and southward conditions. At first glance this is a bit surprising since the spatial morphology of the dayside FAC system is well known to be dependent on the IMF conditions (e.g. Anderson et al., 2008; Korth et al., 2010). However, we must keep in mind that our analysis addresses the spatiotemporal characteristics of the FAC system (i.e. the dynamic stability) not its morphology. Thus, a change in the IMF $B_{\mathrm{Z}}$, either northward of southward, may cause a change in the morphology but apparently not the spatiotemporal characteristics of the FAC system. In contrast, the nightside shows a clear dependence on IMF $B_{\mathrm{Z}}$ orientation. As for the amplitude discussion we argue that this apparent dependence is due to the fact that disturbed times are associated with southward IMF (e.g. Kamide et al., 1977; Kamide and Kokubun, 1996). Indeed we find the median AL to be $-25 \mathrm{nT}$ and $-150 \mathrm{nT}$ for northward and southward IMF conditions, respectively.

In conclusion, we find that the dayside FAC characteristics are independent on IMF $B_{\mathrm{Z}}$ and geomagnetic activity as measured by AL. The nightside FAC system on the other hand is clearly dependent on activity and we argue that the apparent dependence on IMF $B_{\mathrm{Z}}$ is due to the relationship between IMF $B_{\mathrm{Z}}$ and AL. Thus, the plots shown in Figs. 7 and 8 which included all events represent an average ignoring the above mentioned dependencies.

\subsection{Magnetospheric reconfiguration time}

The reconfiguration time of the M-I system is typically stated to be on the order of $10 \mathrm{~min}$ (e.g. Murr and Hughes, 2001) referring to the time it takes the magnetosphere to undergo a large-scale reconfiguration for example from a stretched to a more dipolar configuration. During the reconfiguration energy stored in the magnetosphere is transported along the field lines to the ionosphere where it is dissipated. In this section we provide a possible interpretation of the results but wishes to emphasize that careful simulations and theoretical studies must be performed to confirm and refute our interpretation (these are outside the scope of the current paper).

From Fig. 12, for both dayside and nightside, we find good correlation for small-scale FACs, only if the satellite separation is small. Intuitively it seems appealing that the current 

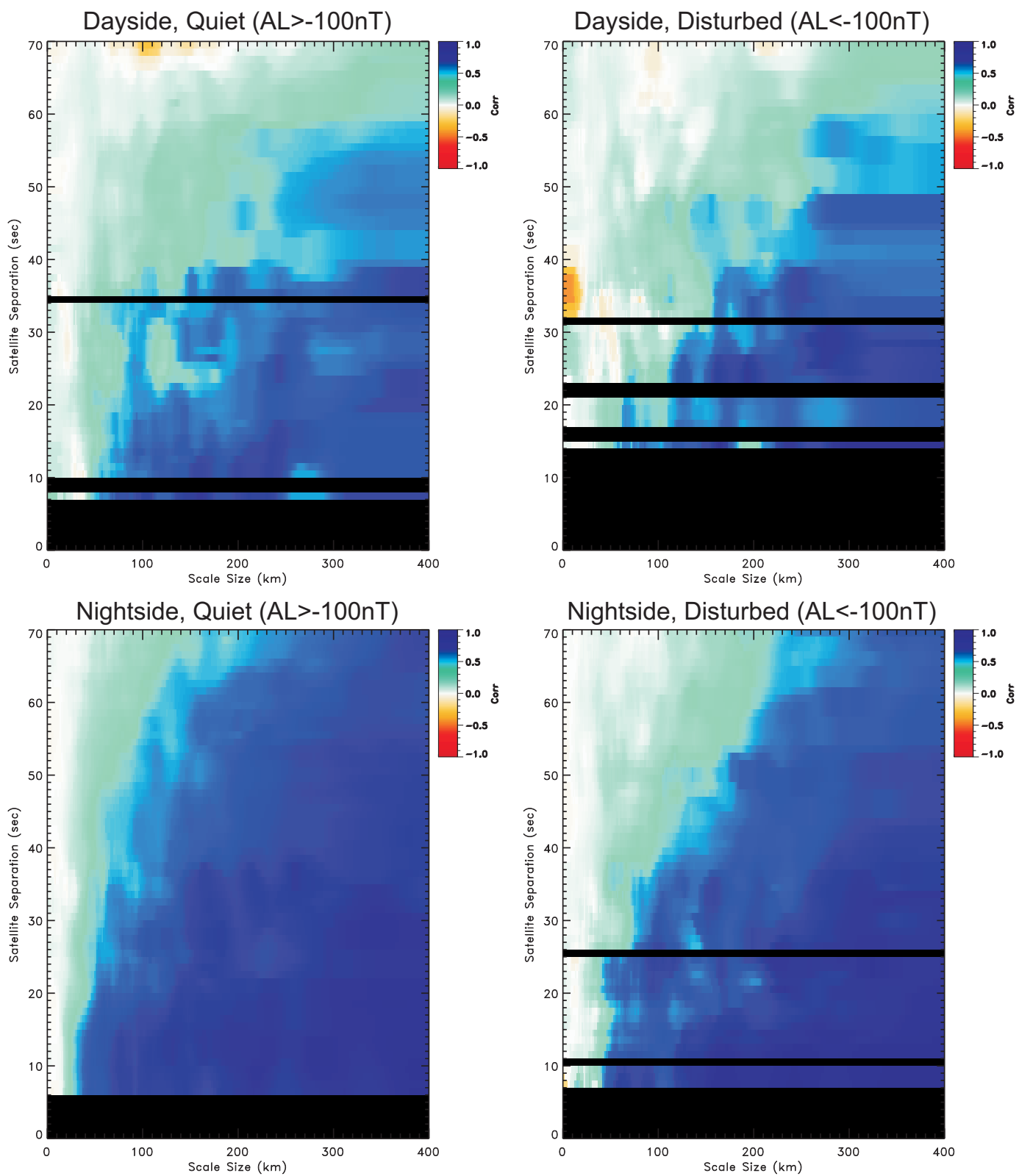

Fig. 13. Correlation for small scale sizes and satellite separations less than $70 \mathrm{~s}$ for dayside and nightside during quiet and disturbed conditions. Note the clear activity dependence on the nightside. The horizontal black bars are due to a lack of events in these intervals.

systems become more stable with increasing scale sizes. Or, in other words, that a $5 \mathrm{~km}$ wide FAC sheet is less stable than a $500 \mathrm{~km}$ wide sheet.

In Figs. 7, 12, 13, and 14 the $\mathrm{X}$-axis (scale size, km) reflects the scale size of the FAC system in the magnetospheric source-region. The Y-axis (satellite separation, s) is the time separation between successive measurements and thus provides information regarding the stability of the FAC system. This, however, comprises the variability of the source region and the Alfvén wave transit time $\left(T_{\mathrm{A}}\right)$ between the source region and the ionosphere where the measurements are taken.
On the dayside we find $T_{\mathrm{A}} \sim 60 \mathrm{~s}$. For $\Delta T<60 \mathrm{~s}$, any changes newly occurred in the source region are never detectable with consecutive orbits at the ionosphere altitudes. For $\Delta T>60 \mathrm{~s}$ the M-I system has enough time to adjust itself to the changed conditions of the solar wind driver and therefore the correlation for such events simply reflect the temporal variation of the solar wind. The calculated correlation thus varies from orbit to orbit as a function of the solar wind driver properties (manifested on the dayside panels, top-left panel of Fig. 12). In this interpretation we can argue that the breakpoint located at $\Delta T \sim 60 \mathrm{~s}$ is the minimum 

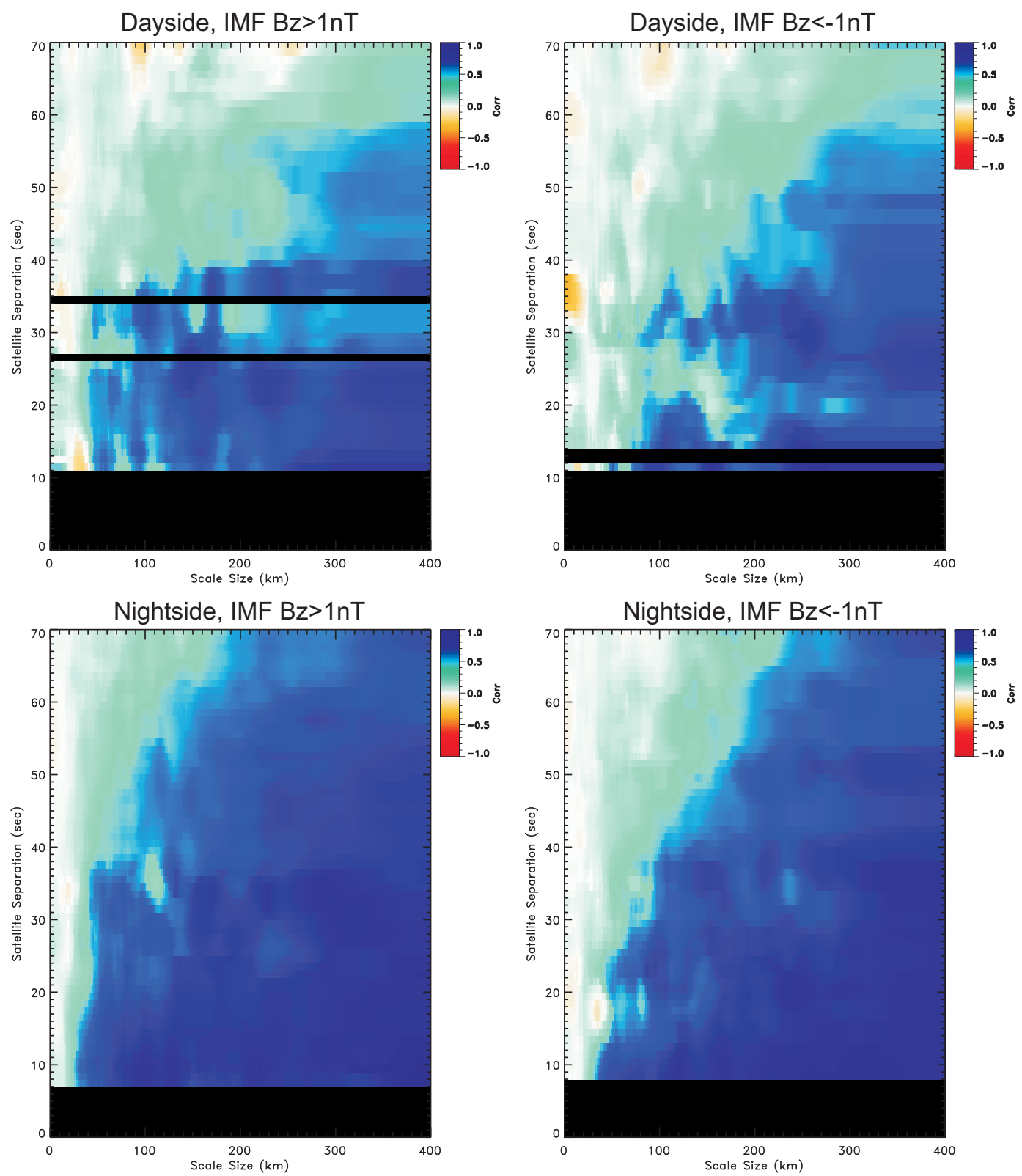

Fig. 14. Same as Fig. 13 but organized by IMF $B_{\mathrm{Z}}$ conditions.

time required for the FAC system to respond to a change in the solar wind driver.

On the nightside we find a somewhat more complex picture. For $\Delta T<160 \mathrm{~s}$, the same argument holds as on the dayside although the field lines are much longer on the nightside and the Alfven transit time is likewise longer $\left(T_{\mathrm{A}} \sim 160 \mathrm{~s}\right)$. For $\Delta T>160 \mathrm{~s}$ another linear relationship is apparent for which the minimum spatial scale with good correlation tends to increase with increasing satellite separation. This is unlike what we find on the dayside where the correlation breaks down for times exceeding the, $\Delta T=T_{\mathrm{A}}$, breakpoint. We interpret this additional linear relationship as being associ- ated with the dynamic properties of the plasma sheet. This interpretation suggests that a second time constant (breakpoint) may exist $\left(T_{\mathrm{p}}\right)$. For $\Delta T$ exceeding the time it takes the plasma sheet to reconfigure, $T_{\mathrm{p}}$, (such as the loading and unloading of energy in the magnetotail) we expect that the FAC characteristics simply reflect variations in the solar wind properties (similar to the dayside for $\Delta T>60 \mathrm{~s}$ ). We may see a glimpse of this region for $\Delta T>600 \mathrm{~s}$ where significant scatter is apparent. Unfortunately, the ST 5 satellite separation range $(<750 \mathrm{~s})$ and event coverage (top panel Fig. 2) does not allow us to perform a robust investigation of this possibility. 

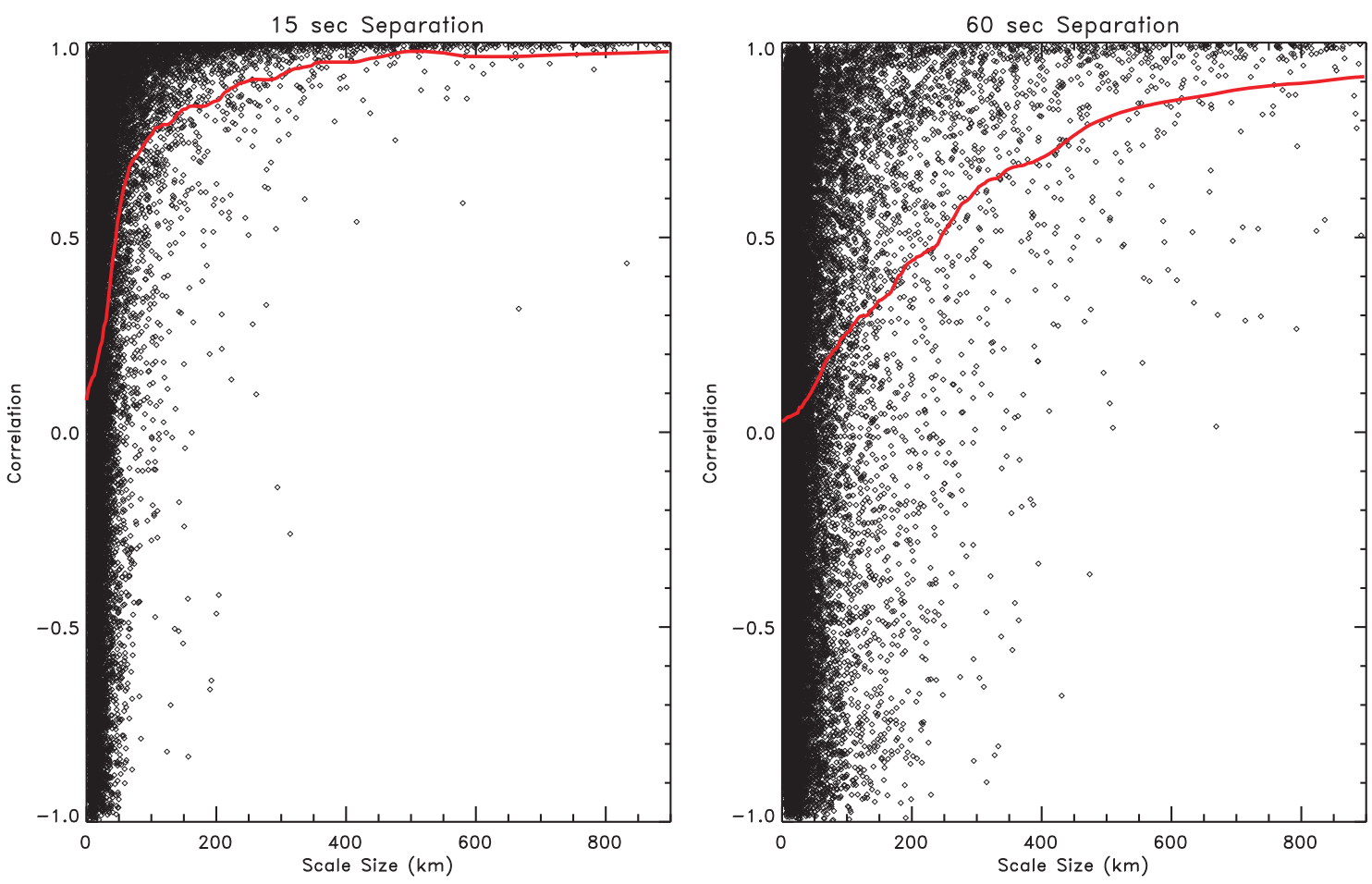

Fig. 15. All data points used in deducing average correlation for two satellite separations. Average values (red lines) are horizontal cross sections of Fig. 7.

\subsection{Robustness of results}

We find that the spatiotemporal characteristics of the FAC system is remarkably constant with systematic differences between dayside and nightside and with a nightside dependence on geomagnetic activity. In the various FAC correlation figures there is no indication of the standard deviation. Some scatter is apparent for satellite separations exceeding $\sim 70$ s when the number of events per s separation falls off sharply thereby providing some information as to the spread of the data. Figure 15 shows all data points contributing to the determination of the average correlation for two given satellite separations using the entire database. Hence, the red lines (averages) are horizontal cross sections of Fig. 7. The systematic behavior shown in Fig. 7 is clearly supported by the individual data points so our average patterns are indeed representative of the typical FAC characteristics.

\subsection{Implications}

The linkage between the magnetosphere and ionosphere in the Earth system has parallels with other planetary and dynamical plasmas. Any system in which forced plasma convection is linked by magnetic lines of force to an ionosphere or similar medium that allows current closure across lines of force will exhibit similar dynamics. The magnetospheres of Jupiter, Saturn and possibly Mercury, as well as the solar corona-photosphere, host systems similar to Earth's magnetotail-ionosphere region. The findings of the present paper has application beyond the terrestrial environment. We find it spectacularly surprising that the characteristics of the FAC system are conserved from event to event. If this was not the case our analysis would not produce well organized regions of high and low correlation but rather a scattered poorly organized pattern. FACs play a key role in the energy and momentum transport between the magnetosphere and ionosphere. The most remarkable finding is that the characteristics of the FAC system appear to be repeatable. That is the magnetosphere use repeatable solutions to shred energy and momentum. We therefore speculate that our results can be generalized to any planetary magnetosphere although the slope and location of the correlated/uncorrelated boundary likely depend on parameters such as the local solar wind conditions, the size of the magnetosphere, and the properties of the load (for example the conductivity matrix of the ionosphere or regolith properties).

Our findings indicate that the transport of energy and momentum between the magnetosphere and the ionosphere is dominated by the large-scale FACs. Not only are these less variable but they are also of larger amplitude. This effectively validates the Zmuda and Armstrong (1974) and Iijima and Potemra (1978) results and assumption that as a statistical average the large scale currents are dominant in the transport of energy and momentum between the magnetosphere and the ionosphere. FACs with smaller scale sizes than the 
R1-R2 currents contribute to a lesser extent than large scale currents but may very well play significant roles during individual events.

Comparisons with previous studies is complicated by the fact that the present study is the first ever comprehensive statistical study of the spatiotemporal characteristics of the field-aligned current system. Single satellite studies of the field-aligned current system typically assume that the measured perturbations are due to static currents regardless of their spatial size. In that respect our results provide a test of the static current hypothesis. One example is the ground breaking studies by Zmuda and Armstrong (1974) and Iijima and Potemra (1978) of the large-scale region 1 and 2 current system. They found these east-west extended current sheets to have latitudinal widths of about 2-3 degrees or 200$300 \mathrm{~km}$. Using the above simple relationships (Sect. 5) significant changes to these current segments occur in roughly $40-60 \mathrm{~s}$ on the dayside and $90-140 \mathrm{~s}$ on the nightside. Assuming a spacecraft velocity of $7 \mathrm{~km} \mathrm{~s}^{-1}$ it takes the satellite $30-40$ s to traverse the sheets. This only marginally supports the static assumption on the dayside. Using rockets travelling at velocities of $\sim 2 \mathrm{~km} \mathrm{~s}^{-1}$, however, would have raised serious questions regarding the static current assumption on both the nightside and the dayside. Instrumentation making measurements of an ionospheric electrodynamic parameter with a $60 \mathrm{~s}$ integration will not be able to resolve features with scale sizes smaller than $100-300 \mathrm{~km}$ depending on local time. Naturally, this assumes that our findings of the FAC characteristics can be generalized to other electrodynamic parameters such as plasma convection and electrical conductances. An assumptions which seems reasonable since these are coupled through fundamental equations such as current continuity and Ohm's law. Observations of precipitating particles made by the two Dynamics Explorer spacecraft support the above generalization (Thieman and Hoffman, 1985). They investigated 28 perigee passes when the separation of the two spacecraft was within $18 \mathrm{~min}$. They found that a few minutes could result in significant differences in the maximum energy of inverted $\mathrm{V}$ events in good agreement with our results.

\section{Summary and conclusions}

We presented the first ever comprehensive statistical study of the spatiotemporal characteristics of field-aligned currents in the terrestrial magnetosphere-ionosphere system using multi point measurements. We determined how the density, the variability and the scale size are coupled. The three ST 5 satellites were in a pearls-on-a-string formation making measurements of the magnetic field with variable inter-spacecraft separations ranging from a few seconds to about $10 \mathrm{~min}$. We identified sets of satellite passes for which both satellites provide magnetic field measurements during the auroral crossings and found more than 4700 such events. These were analyzed using a robust correlation analysis aimed at determining the variability of the FAC system as a function of scale size and satellite spacing. Our findings indicate that the magnetosphere-ionosphere system is a repeatable system for which the FAC characteristics are maintained from event to event. We found significant differences between the FAC characteristics on the dayside and on the nightside. On the dayside the FAC characteristics are found to be independent of IMF $B_{\mathrm{Z}}$ and geomagnetic activity while the nightside indicate increased variability during disturbed conditions. The boundary separating highly and poorly correlated FACs can be fitted by a linear line for satellite separations shorter than $60 \mathrm{~s}$ (dayside) and $160 \mathrm{~s}$ (nightside). We interpret this as the dayside and nightside magnetospheric reconfiguration time, respectively. For time scales exceeding this we suggest the FAC characteristics are controlled by the solar wind (dayside) and plasma sheet (nightside) dynamics. Finally, the characteristics of FAC system with scale sizes larger than $\sim 200 \mathrm{~km}$ (at ionospheric altitude) appear to be stable and repeatable on time scales of the order of a minute (i.e. comparable to the low-altitude orbiting satellite's traverse time across the auroral belt). In this sense, our results effectively validate the Iijima and Potemra (1978) assumption that on average the large-scale currents with scale sizes of the Region 1 and Region 2 are quasi-persistently and significant in the transport of energy and momentum between the magnetosphere and the ionosphere.

Acknowledgements. We gratefully acknowledge the helpful comments by K. Takahashi, R. A. Hoffman, and A. T. Y. Lui. We gratefully acknowledge the ST 5 magnetometer team: R. Strangeway, G. Le and J. Slavin. The first author acknowledges NASA contract NNX08AM32G.

Topical Editor R. Nakamura thanks two anonymous referees for their help in evaluating this paper.

\section{References}

Anderson, H. R. and Vondrak, R. R.: Observations of Birkeland currents at auroral latitudes, Rev. Geophys. Space. Phys., 13, 243262, 1975.

Anderson, B. J., Takahashi, K., and Toth, B. A.: Sensing global Birkeland currents with Iridium engineering magnetometer data, Geophys. Res. Lett., 27, 4045-4048, 2000.

Anderson, B. J., Korth, H., Waters, C. L., Green, D. L., and Stauning, P.: Statistical Birkeland current distributions from magnetic field observations by the Iridium constellation, Ann. Geophys., 26, 671-687, doi:10.5194/angeo-26-671-2008, 2008.

Birkeland, K.: Norwegian Aurora Polaris Expedition, 1902-03, vol. 1, 1st sec., Aschhough \& Co, Christiania, 1908.

Christiansen, F., Papitashvili, V. O., and Neubert, T.: Seasonal variations of high-latitude field-aligned currents inferred from Ørsted and Magsat observations, J. Geophys. Res., 107, 1029, doi:10.1029/2001JA900104, 2002.

Davis, T. N. and Sugiura, M.: Auroral electrojet activity index AE and its universal time variations, J. Geophys. Res., 71, 785-801, 1966. 
Escoubet, C. P., Fehringer, M., and Goldstein, M.: Introduction: The Cluster mission, Ann. Geophys., 19, 1197-1200, doi:10.5194/angeo-19-1197-2001, 2001.

Foster, J. C., St.-Maurice, J.-P., and Abreu, V. J.: Joule heating at high latitudes, J. Geophys. Res., 88, 4885-4896, 1983.

Fujii, R. and Iijima, T.: Control of the ionospheric conductivities on large-scale Birkeland current intensities under geomagnetic quiet conditions, J. Geophys. Res., 92, 4505-4513, 1987.

Hoffman, R. A., Fujii, R., and Sugiura, M.: Characteristics of the field-aligned current system in the nighttime sector during auroral substorms, J. Geophys. Res., 99, 21303-21325, 1994.

Iijima, T.: Field-aligned currents in geospace: Substance and significance, in: Magnetospheric Current Systems, Geophys. Monograph. Ser., vol. 28, edited by: Ohtani, S.-I., Fujii, R., Hesse, M., and Lysak, R. L., 107, AGU, Washington, D.C., 2000.

Iijima, T. and Potemra, T. A.: The amplitude distribution of fieldaligned currents at northern high latitudes observed by Triad, J. Geophys. Res., 81, 2165-2174, 1976.

Iijima, T. and Potemra, T. A.: Large-scale characteristics of fieldaligned currents associated with substorms, J. Geophys. Res., 83, 599-615, 1978.

Juusola, L., Kauristie, K., Amm, O., and Ritter, P.: Statistical dependence of auroral ionospheric currents on solar wind and geomagnetic parameters from 5 years of CHAMP satellite data, Ann. Geophys., 27, 1005-1017, doi:10.5194/angeo-27-10052009, 2009.

Kamide, Y:: The relationship between field-aligned currents and the auroral electrojets: A review, Space. Sci. Rev., 31, 127-243, 1982.

Kamide, Y. and Kokubun, S.: Two-component auroral electrojet: Importance for substorm studies, J. Geophys. Res., 101, 1302713046, 1996.

Kamide, Y., Perreault, P. D., Akasofu, S.-I., and Winningham, J. D.: Dependence of Substorm Occurrence Probability on the Interplanetary Magnetic Field and on the Size of the Auroral Oval, J. Geophys. Res., 82, 5521-5528, 1977.

Korth, H., Anderson, B. J., and Waters, C. L.: Statistical analysis of the dependence of large-scale Birkeland currents on solar wind parameters, Ann. Geophys., 28, 515-530, doi:10.5194/angeo28-515-2010, 2010.

Le, G., Wang, Y., Slavin, J. A., and Strangeway, R. J.: Space Technology 5 multipoint observations of temporal and spatial variability of field-aligned currents, J. Geophys. Res., 114, A08206, doi:10.1029/2009JA014081, 2009.

Lu, G., Baker, D. N., McPherron, R. L., Farrugia, C. J., Lummerzheim, D., Ruohoniem, J. M., Rich, F. J., Evans, D. S., Lepping, R. P., Brittnacher, M., Li, X., Greenwald, R., Sofko, G., Villain, J., Lester, M., Thayer, J., Moretto, T., Milling, D., Troshichev, O., Zaitzev, A., Odintzov, V., Makarov, G., and Hayashi, K.: Global energy deposition during the January 1997 magnetic cloud event, J. Geophys. Res., 103, 11685-11694, 1998.

Luhr, H., Warnecke, J., Zanetti, L., Lindqvist, P. A., and Hughes, T. J.: Fine structure of field-aligned current sheets deduced from spacecraft and ground-based observations: Initial Freja results, Geophys. Res. Lett., 21, 1883-1886, 1994.
Lynch, K. A., Pietrowski, D., Torbert, R. B., Ivchenko, N., Marklund, G., and Primdahl, F.: Multiple-point electron measurements in a nightside auroral arc: Auroral Turbulence II particle observations, Geophys. Res. Lett., 26, 3361-3364, 1999.

Murr, D. L. and Hughes, W. J.: Reconfiguration timescales of ionospheric convection, Geophys. Res. Lett., 28, 2145-2148, 2001.

Ohtani, S., Blomberg, L. G., Newell, P. T., Yamauchi, M., Potemra, T. A., and Zanetti, L. J.: Altitudinal comparison of dayside field-aligned current signatures by Viking and DMSP-F7: Intermediate-scale field-aligned current systems, J. Geophys. Res., 101, 15297-15310, 1996.

Rostoker, G.: Geomagnetic indices, Rev. Geophys. Space Phys., 10, 935-950, 1972.

Rostoker, G., Armstrong, J. C., and Zmuda, A. J.: Field-aligned current flow associated with intrusion of the substorm-intensified westward electrojet into the evening sector, J. Geophys. Res., 80, 3571-3579, 1975.

Slavin, J. A., Le, G., Strangeway, R. J., Wang, Y., Boardsen, S. A., Moldwin, M. B., and Spence, H. E.: Space Technology 5 multipoint measurements of near-Earth magnetic fields: Initial results, Geophys. Res. Lett., 35, L02107, doi:10.1029/2007GL031728, 2008.

Song, P. and Russell, C. T.: Time series data analyses in space physics, Space Sci. Rev., 87, 387-463, 1999.

Sugiura, M.: A fundamental magnetosphere-ionosphere coupling mode involving field-aligned currents as deduced from DE-2 observations, Geophys. Res. Lett., 11, 877-880, 1984.

Thieman, J. R. and Hoffman, R. A.: Determination of inverted-V stability from Dynamics Explorer satellite data, J. Geophys. Res., 90, 3511-3516, 1985.

Weimer, D. R.: Correction to "Predicting interplanetary magnetic field (IMF) propagation delay times using the minimum variance technique," J. Geophys. Res., 109, A12104, doi:10.1029/2004JA010691, 2004.

Weimer, D. R., Ober, D. M., Maynard, N. C., Collier, M. R., McComas, D. J., Ness, N. F., Smith, C. W., and Watermann, J.: Predicting interplanetary magnetic field (IMF) propagation delay times using the minimum variance technique, J. Geophys. Res., 108, doi:10.1029/2002JA009405, 2003.

Zheng, Y., Lynch, K. A., Boehm, M., Goldstein, R., Javadi, H., Schuck, P., Arnoldy, R. L., and Kintner, P. M.: Multipoint measurements of field-aligned current density in the auroral zone, J. Geophys. Res., 108, 1217, doi:10.1029/2002JA009450, 2003.

Zmuda, A. J. and Armstrong, J. C.: The diurnal flow pattern of field-aligned currents, J. Geophys. Res., 79, 4611-4619, 1974.

Zmuda, A. J., Martin, J. H., and Heuring, F. T.: Transverse magnetic disturbances at 1100 kilometers in the auroral region, J. Geophys. Res., 71, 5033-5045, 1966.

Zmuda, A. J., Heuring, F. T., and Martin, J. H.: Dayside magnetic disturbances at 1100 kilometers in the auroral oval, J. Geophys. Res., 72, 1115-1117, 1967. 